\title{
Embolectomía pulmonar quirúrgica en el manejo del tromboembolismo pulmonar agudo: reporte de caso
}

\author{
Diego Humberto Márquez-Bohórquez* \\ Jaime Calderón-Herrera**
}

\begin{abstract}
*Residente de IV Año de Cirugía General. Universidad Industrial de Santander. Bucaramanga. Santander. Colombia.
**Cirujano Cardiovascular. Profesor de Cirugía General. Universidad Industrial de Santander. Bucaramanga. Santander. Colombia. Correspondencia: Dr. Diego Humberto Márquez B. Dirección: Carrera 28 \# 47-47. Apartamento 503. Edificio Domus Aurea. Bucaramanga. Santander. Colombia. Correo electrónico: diegohmarquezb@gmail.com
\end{abstract}

\section{RESUMEN}

Introducción: El tromboembolismo pulmonar es una patología que compromete la vida y requiere tratamiento inmediato. De esta manera, para obtener los mejores resultados debe existir un alto grado de sospecha clínica que permita realizar el diagnóstico de manera temprana. Su manejo es farmacológico con anticoagulación, pero en ciertas ocasiones requiere de tratamiento quirúrgico. El objetivo de este reporte de caso es describir las indicaciones actuales para embolectomía pulmonar quirúrgica en un paciente con tromboembolismo pulmonar. Presentación del caso: Se presenta un caso de una paciente con tromboembolismo pulmonar, inestabilidad hemodinámica y foramen oval permeable, en el cual se encontraba atrapado un trombo en silla de montar con extensión a las dos aurículas, quien fue tratada con embolectomía pulmonar quirúrgica. Posterior a la embolectomía pulmonar quirúrgica la paciente presentó un episodio de tromboembolismo pulmonar recurrente que fue manejado nuevamente con embolectomía quirúrgica previo a la implantación de un filtro de vena cava por vía percutánea. Su evolución fue satisfactoria permitiendo suspender el soporte ventilatorio y hemodinámico de manera temprana. Conclusiones: La embolectomía pulmonar quirúrgica se mantiene vigente como manejo del tromboembolismo pulmonar en pacientes con inestabilidad hemodinámica que tienen contraindicación para trombolisis o en quiénes esta terapia ha fallado, así como en pacientes que tienen un trombo intracardíaco o en un foramen oval permeable. MÉD.UIS. 2017;30(1):93-7.

Palabras clave: Embolía Pulmonar. Embolectomía. Foramen Oval Permeable. Trombosis Venosa.

\section{Surgical pulmonary embolectomy for the management of acute pulmonary embolism: a case report}

\section{ABSTRACT}

Introduction: The pulmonary embolism is a pathology that endangers life and it requires an immediate treatment. In order to get the best results there should be a high degree of clinical suspicion that allows us to make an early diagnosis. It requires a pharmacological treatment with anticoagulation but in certain cases there is necessary a surgical treatment. The objective of this case report is to describe the current indications for the surgical pulmonary embolectomy in a patient with pulmonary embolism. Case Presentation: This is the presentation of a case in a patient with pulmonary embolism, haemodynamic instability and a horse saddle thrombus located in a patent foramen ovale which was expanded on the both heart atriums. This patient was treated with surgical pulmonary embolectomy. Following the surgical pulmonary embolectomy the patient had a recurrent pulmonary embolism episode that was treated with the insertion of a percutaneous vena cava filter and a newly surgical pulmonary embolectomy. The patient had a satisfactory postoperative evolution with an early discontinuation of the ventilatory and haemodynamic support. Conclusion: The surgical pulmonary embolectomy is still an applicable treatment for the pulmonary embolism in patients with haemodynamic instability, absolute contraindication to systemic thrombolytic therapy or failure to this therapy, patent foramen ovale and an intracardiac floating thrombus. MÉD.UIS. 2017;30(1):93-7.

Keywords: Pulmonary embolism. Embolectomy. Foramen oval, Patent. Venous thrombosis.

Artículo recibido el 1 de Febrero de 2016 y aceptado para publicación el 20 de Enero de 2017 
¿Cómo citar este artículo?: Márquez-Bohórquez DH, Calderón-Herrera J. Embolectomía pulmonar quirúrgica en el manejo del tromboembolismo pulmonar agudo: reporte de caso. MÉD.UIS. 2017;30(1):93-7.

\section{INTRODUCCIÓN}

El Tomboembolismo Pulmonar (TEP) es la causa más común de muerte prevenible en pacientes hospitalizados. En Estados Unidos se ha reportado que la mortalidad varía entre 15 al $30 \%$ en dichos pacientes'. Siendo el Troemboembolismo Venoso (TEV) el desencadenante mas importante, se ha registrado que la incidencia de TEV en cirugía mayor, sin profilaxis antitrombótica, alcanza el $25 \%$ y puede ser tan alta como el $60 \%$ en trauma mayor y $90 \%$ en lesiones de la médula espinal'. Colombia participó en el Registro Internacional sobre Prevención Médica del TEV (IMPROVE) donde se evidenció que en los centros colombianos participantes se hizo mayor profilaxis contra TEV en pacientes apropiados, a comparación con el resto de paises. Teniendo en cuenta la importancia del TEP es vital reconocer que el resultado final depende del tamaño del trombo, la función cardiopulmonar de base y la prontitud del diagnóstico e instauración del tratamiento. De acuerdo a esto, se debe sospechar en casos de hipoxemia, hipotensión y compromiso hemodinámico.

La incidencia del TEP en pacientes hospitalizados es del $25 \%$, donde se tiene como opciones terapéuticas el manejo médico con anticoagulación y trombolisis, así como quirúrgicas con la embolectomía pulmonar. Al comparar la mortalidad del tratamiento con trombolisis $(9,84 \%)$ con la mortalidad de la embolectomía pulmonar quirúrgica (3,33\%) se obtuvo que las complicaciones por sangrado son mayores con la trombolisis, donde se ha descrito hemorragias a nivel cerebral y gastrointestinal las cuales pueden ser fatales ${ }^{8}$

Por medio de este reporte de caso se pretende resaltar las indicaciones actuales de embolectomía pulmonar quirúrgica en el paciente con TEP agudo.

\section{FACTORES DE RIESGO}

La base principal para el desarrollo de TEP es el TEV, el cual se fundamenta en la tríada de Virchow, la cual consiste en estasis, lesión del vaso e hipercoagulabilidad³. La estasis venosa puede verse en pacientes con inmovilidad y falla cardíaca. En cuanto a la lesión del vaso, esta se evidencia en pacientes con antecedente de cirugía o trauma. Por otro lado, la hipercoagulabilidad se observa en pacientes con cáncer, medicamentos, anticonceptivos orales y con herencia de síndromes protrombóticos como resistencia a la proteína $C$ activada, deficiencia de proteína $\mathrm{C}$ y $\mathrm{S}$, mutaciones de la protrombina y elevación de la homocisteína. Otros factores de riesgo incluyen la ventilación mecánica invasiva, el uso de bloqueadores neuromusculares, la presencia de catéteres venosos centrales, la insuficiencia renal terminal y el embarazo ${ }^{1,4}$.

\section{Fisiopatología}

La mayoría (80-90\%) de los trombos se originan de la trombosis en las venas profundas de las extremidades inferiores ${ }^{5}$. Por tanto, el TEP ocurre secundario a la obstrucción mecánica del flujo sanguíneo en la arteria pulmonar por émbolos provenientes de dichas venas y la respuesta humoral desencadenada. El émbolo se sitúa en la arteria pulmonar, produciendo una activación de las plaquetas y liberación de agentes vasoactivos, como la histamina, el complemento, el tromboxano y la serotonina, los cuales incrementan la resistencia vascular pulmonar'. Debido a esto se desencadena un aumento del volumen telediastólico del ventrículo derecho, lo cual lleva a dilatación del mismo y desplazamiento del septo interventricular. Seguido de esto se da una disminución del llenado en el ventrículo izquierdo y el flujo coronario dando como resultado una disfunción cardíaca global, colapso hemodinámico e hipoperfusión sistémica ${ }^{3}$. No obstante, la presencia de un foramen oval permeable empeora el cuadro secundario al shunt intracardíaco, el cual exacerba la hipoxia y la vasoconstricción pulmonar ${ }^{8}$.

\section{OpCIONES TERAPÉUTICAS}

Dentro de las opciones de tratamiento para el TEP se cuenta con la anticoagulación, la trombolisis sistémica, la embolectomía pulmonar por catéter o la trombolisis dirigida por catéter, y la embolectomía pulmonar quirúrgica. La anticoagulación como única 
medida de manejo está indicada en los pacientes estables hemodinámicamente que tienen función ventricular derecha normal. La trombolisis sistémica está indicada en pacientes que tienen evidencia de disfunción ventricular derecha. Los tratamientos con catéter, como la embolectomía y trombolisis, se consideran en casos donde falla la trombolisis sistémica y cuando la embolectomía quirúrgica no es posible, como es el caso de los pacientes que se encuentran hospitalizados en centros donde no se cuenta con la disponibilidad de circulación extracorpórea. Por último, la embolectomía pulmonar quirúrgica debe ser implementada cuando las anteriores técnicas son fallidas o en los pacientes donde no hay suficiente tiempo para realizar una terapia trombolítica efectiva ${ }^{1,9}$.

La embolectomía pulmonar quirúrgica consiste en la extracción del trombo a través de una arteriotomía en la arteria pulmonar, previa esternotomía. Esta tecnica debe considerarse en los pacientes con trombo intracardíaco o complicaciones sistémicas embólicas secundarias a un foramen oval permeable u otros defectos septales', así como la presencia de refractariedad al manejo médico con anticoagulación, contraindicación para trombolisis o insuficiente tiempo para realizar una trombolisis efectiva. Es importante conocer esta técnica dado que los estudios han demostrado que la embolectomía pulmonar quirúrgica tiene resultados superiores cuando se compara con la trombolisis a repetición ${ }^{11}$ (Ver Figura 1).

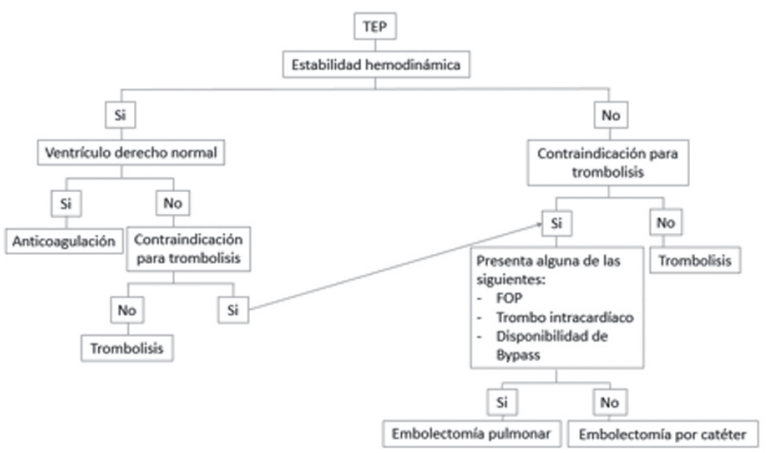

Figura 1. Algoritmo terapéutico para el manejo del tromboembolismo pulmonar agudo(3)(2. FOP: Foramen Oval Permeable. TEP: Tromboembolismo pulmonar.

Fuente: autor.

\section{Presentación del caso}

Paciente de 64 años, hospitalizada en la Clínica FOSCAL Internacional de la ciudad de Floridablanca, Santander, sexo femenino, con antecedente de hipertensión arterial, enfermedad renal crónica e hipotiroidismo, quien presentó disnea de aparición súbita. Se realizó angiotomografía pulmonar y se documentó TEP con un trombo en la arteria lobar derecha (Ver Figuras 2 y 3). Además se realizó un ecocardiograma transtorácico que mostró trombo en silla de montar con extensión a las dos aurículas a través de un foramen oval permeable. Se realizó esternotomía mediana, disección por planos hasta ingresar a cavidad pericárdica, canulación aortocava, arteriotomía pulmonar y aspiración de los trombos localizados en las ramas pulmonares por medio de aspiración directa con cánula de succión. Luego se hizo una atriotomía derecha extrayendo un coágulo de $6 \mathrm{~cm}$ de longitud y se amplió el defecto del septo interauricular retirando un coágulo de $5 \mathrm{~cm}$ de longitud. Posteriormente, se realizó el cierre de la comunicación interauricular con un parche de pericardio autólogo. A través del ventrículo derecho se introdujo un aspirador al árbol pulmonar logrando extraer otro trombo de $3 \mathrm{~cm}$ de longitud (Ver Figura 4). A las 48 horas la paciente presentó un episodio de TEP recurrente requiriendo una nueva intervención quirúrgica. De esta manera, como medida de control al TEP recurrente se implantó un filtro de vena por vía percutánea para luego realizar la arteriotomía pulmonar longitudinal, logrando extraer tres coágulos más de las ramas pulmonares. La paciente evolucionó satisfactoriamente en su recuperación postoperatoria permitiendo suspender el soporte ventilatorio y hemodinámico de manera temprana en la Unidad de Cuidado Intensivo. Posteriormente, se trasladó a piso donde no presentó complicaciones dándose egreso a las tres semanas de su ingreso a urgencias. Acudió al control por consulta externa un mes después sin presentar complicaciones cardiopulmonares.

\section{Discusión}

Este caso clínico de TEP es de vital importancia e interés dado que nos remonta a la situación donde el manejo médico de esta entidad no es suficiente, teniendo que recurrir al abordaje quirúrgico como medida terapéutica. La paciente del presente caso, además de presentar TEP, tuvo un trombo localizado en el foramen oval permeable, hallazgo que indicó la embolectomía pulmonar quirúrgica como única alternativa de tratamiento. En todos los estudios realizados en los últimos años se recomienda el abordaje quirúrgico para aquellos pacientes con trombo intra-cardíaco o en un foramen oval 
permeable, en comparación con la anticoagulación y trombolisis, incluso, se resaltan los resultados superiores de la cirugía en comparación con la trombolisis a repetición ${ }^{12,13}$. Al paciente con TEP que presenta inestabilidad hemodinámica se le debe valorar la función ventricular derecha con un ecocardiograma. De esta manera, el abordaje médico inicial en estos pacientes es con heparina no fraccionada, la cual brinda la ventaja de poder monitorizar su acción estrictamente. Existe la opción de tratamiento médico con heparina de bajo peso molecular, inhibiendo el factor $\mathrm{Xa}$, sin embargo, esta heparina no se puede vigilar y revertir de manera urgente en comparación con la heparina sódica. Según los hallazgos ecocardiográficos se definirá la indicación de trombolisis, embolectomía pulmonar quirúrgica o embolectomía por catéter. Adicionalmente, todo paciente inestable hemodinámicamente requiere trombolisis si no presenta ninguna contraindicación para la misma7, por el contrario, si se presenta contraindicación para trombolisis debe ser llevado a una embolectomía pulmonar quirúrgica. Es contraindicación absoluta de la embolectomía pulmonar quirúrgica el hecho que el paciente pueda ser sometido a trombolisis si no tiene un trombo intra-cardíaco o a través de un foramen oval permeable. Es por esto que la gran mayoría de los pacientes con TEP no requieren una embolectomía pulmonar quirúrgica y es indispensable, por lo tanto, tener claras las indicaciones mencionadas previamente.

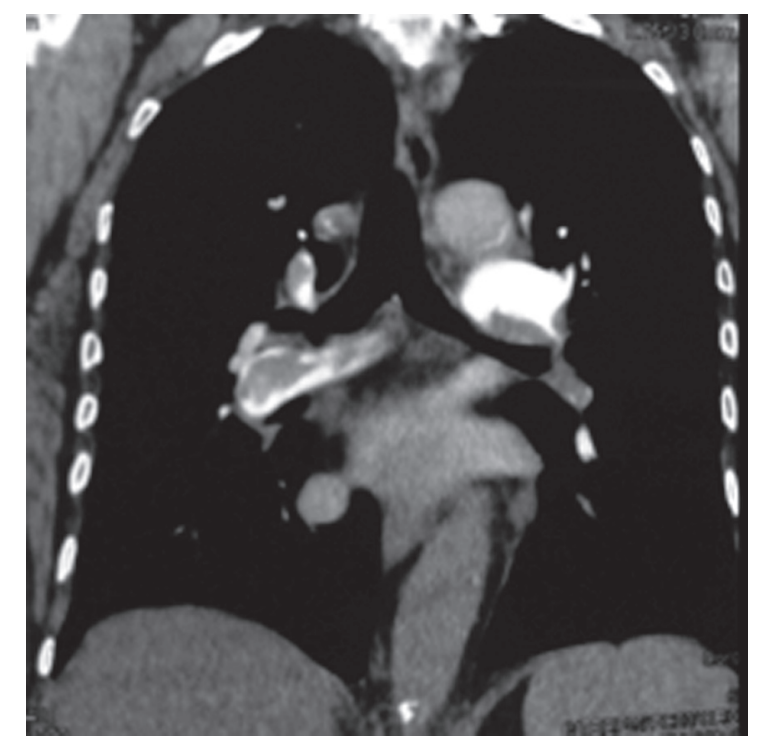

Figura 2. Angiotomografía de tórax con trombo en la arteria lobar derecha, corte coronal.

Fuente: FOSCAL Internacional.

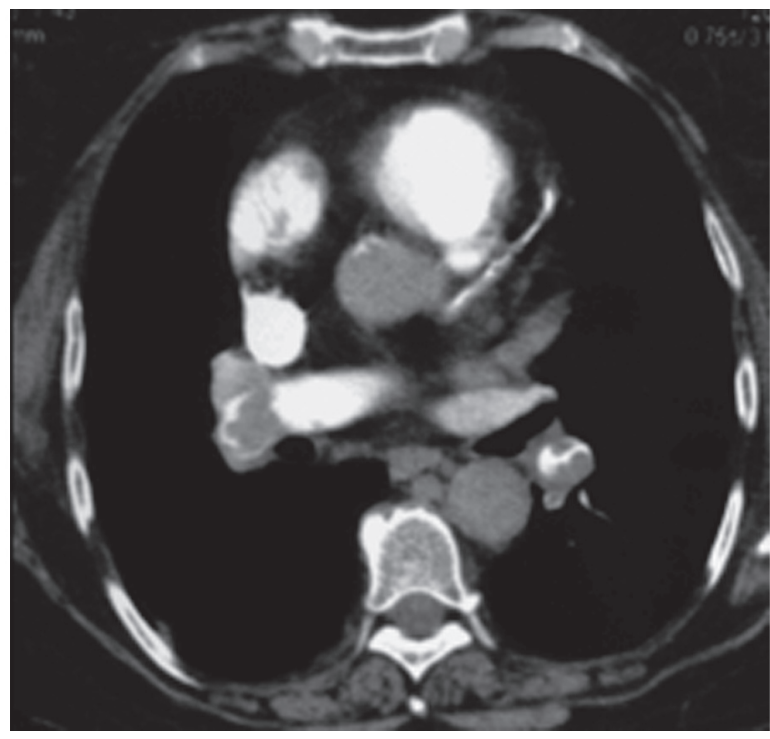

Figura 3. Angiotomografía de tórax con trombo en la arteria lobar derecha, corte axial.

Fuente: FOSCAL Internacional.

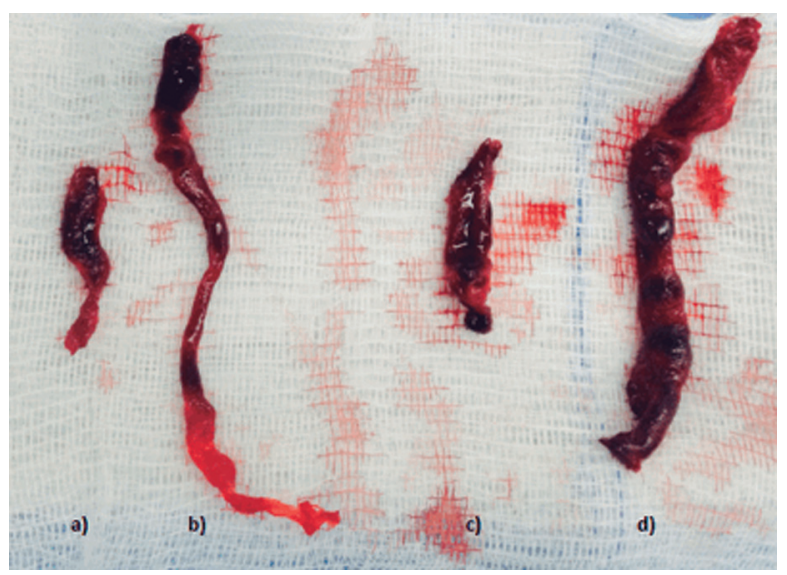

Figura 4. a) Trombo localizado en arteria lobar derecha, b) trombo localizado a través del FOP, c y d) trombos localizados en árbol pulmonar. FOP: foramen oval permeable.

Fuente: autor.

\section{Conclusión}

La embolectomía pulmonar quirúrgica tiene un importante impacto en la mortalidad del TEP agudo comparado con la terapia de trombolisis sistémica y anticoagulación cuando los pacientes presentan inestabilidad hemodinámica asociada refractariedad de la trombolisis, contraindicación a la misma, o si presentan un trombo intracardíaco o a través de un foramen oval permeable. Este abordaje quirúrgico incluso tiene menores tasas de mortalidad comparado con la trombolisis a repetición en este tipo de pacientes. 


\section{CONFLICTO DE INTERESES}

No existe conflicto de intereses entre los autores, el contenido del artículo ni empresas relacionadas o no con la salud.

\section{FinanCiación}

\section{No hubo ningún apoyo financiero.}

\section{Referencias Bibliográficas}

1. Cox JC, Jablons DM. Operative and Perioperative Pulmonary Emboli. Thorac Surg Clin. 2015; 25(3): 289-99.

2. Dennis RJ, Acevedo JR, Restrepo HF, Hernández JI, Rivas E, Sabogal JE. ¿Es apropiada la profilaxis actual del tromboembolismo venoso en pacientes médicos?: Evaluación de desenlaces colombianos en el Registro Internacional sobre Prevención Médica del TEV (IMPROVE). Acta Med Colomb. 2009;34:11-6.

3. Cohn LH. Cardiac surgery in the adult. 4th ed. Boston, Massachusetts: McGraw Hill;2012.

4. Colombier S, Niclauss L. Successful Surgical Pulmonary Embolectomy for Massive Perinatal Embolism after Emergency Cesarean Section. Ann Vasc Surg. 2015;29(7):1452.e1-4.
5. Wu MY, Liu YC, Tseng YH, Chang YS, Lin PJ, Wu TI. Pulmonary embolectomy in high-risk acute pulmonary embolism: The effectiveness of a comprehensive therapeutic algorithm including extracorporeal life support. Resuscitation. 2013;84(10):1365-70.

6. Visnjevac O, Lee K, Bulatovic R, et al. Outcomes-based systematic review for management of massive intra-cardiac or pulmonary thrombotic emboli during surgery. Heart Lung Vessel. 2014;6(1):24-32.

7. Kilic A, Shah As, Conte J V, Yuh DD. Nationwide outcomes of surgical embolectomy for acute pulmonary embolism. J Thorac Cardiovasc Surg. 2013;145(2):373-7.

8. Azari A, Beheshti AT, Moravvej Z, Bigdelu L, Salehi M. Surgical embolectomy versus thrombolytic therapy in the management of acute massive pulmonary embolism: short and long-term prognosis. Heart Lung. 2015;44(4):335-9.

9. Osborne ZJ, Rossi P, Aucar J, Dharamsy S, Cook S, Wheatley B. Surgical pulmonary embolectomy in a community hospital. Am J Surg. 2014;207(3):337-41

10. Fukuda I, Taniguchi S, Fukui K, Minakawa M, Daitoku K, Suzuki Y. Improved outcome of surgical pulmonary embolectomy by aggressive intervention for critically ill patients. Ann Thorac Surg. 2011;91(3):728-32.

11. Meneveau N, Seronde MF, Blonde MC, Legalery P, Didier-Petit $\mathrm{K}$, Briand $\mathrm{F}$, et al. Management of unsuccessful thrombolysis in acute massive pulmonary embolism. Chest. 2006;129:104350 .

12. Takahashi H, Okada K, Matsumori M, Kano H, Okita Y. Aggressive surgical treatment of acute pulmonary embolism with circulatory collapse. Ann Thorac Surg. 2012;94:785-91.

13. Stein PD, Alnas M, Beemath A, Patel NR. Outcome of pulmonary embolectomy. Am J Cardiol. 2007;99(3):421-3. 